\title{
Managing Sexual Dysfunction For Women With Breast Cancer: The Perspective Of Health Care Providers In North East Malaysia.
}

\section{Siti Balqis Chanmekun}

Universiti Sains Malaysia - Kampus Kesihatan

Maryam Mohd Zulkifli

Universiti Sains Malaysia - Kampus Kesihatan

Rosediani Muhamad

Universiti Sains Malaysia - Kampus Kesihatan

Norhasmah Mohd Zain ( $\square$ hasmahmz@usm.my )

Universiti Sains Malaysia - Kampus Kesihatan https://orcid.org/0000-0002-6297-8077

Wah Yun Low

University of Malaya Faculty of Medicine

Pranee Liamputtong

Western Sydney University

\section{Research Article}

Keywords: Breast Cancer, Female Sexual Dysfunction, Health Care Providers, Sexuality, Qualitative Study

Posted Date: March 10th, 2021

DOI: https://doi.org/10.21203/rs.3.rs-161097/v1

License: (c) (i) This work is licensed under a Creative Commons Attribution 4.0 International License.

Read Full License

Version of Record: A version of this preprint was published at Supportive Care in Cancer on July 23rd, 2021. See the published version at https://doi.org/10.1007/s00520-021-06417-0. 


\section{Abstract}

Purpose: Management of female sexual dysfunction (FSD) is vital for women with breast cancer due to the devastating consequences, which include marital disharmony and reduced quality of life. We explore healthcare providers' (HCPs) perceptions and experiences in managing FSD for women living with breast cancer using phenomenological approach.

Methods: This qualitative study was conducted using a face-to-face interview method to HCPs from two tertiary hospitals in North East Malaysia. The interviews were recorded, transcribed verbatim, and transferred to NVivo ${ }^{\circledR}$ for data management. The transcriptions were analyzed using thematic analysis.

Results: Three key barriers were identified through the thematic analysis: a scarcity of related knowledge; the influence of socio-cultural ideas about sex; and the speciality-centric nature of the healthcare system. Most HCPs interviewed had a very narrow understanding of sexuality, were unfamiliar with the meaning of FSD, and felt their training on sexual health issues to be very limited. They viewed talking about sex to be embarrassing to both parties that is, both to HCPs and patients and was therefore not a priority. They focused more on their specialty hence limited the time to discuss sexual health and FSD with their patients.

Conclusion: Therefore, interventions to empower the knowledge, break the sociocultural barriers and improve the clinic settings are crucial for HCPs in managing FSD confidently.

\section{Introduction}

A retrospective cohort study of 10, 230 Malaysian women with breast cancer reported that women younger than 50 had significantly better survival rates than women who were 50 years or older. ${ }^{1}$ Sexual health in cancer patients is an indicator to quality of life but it is infrequently assessed by the healthcare providers (HCPs) and should be evaluated at each clinic visit. ${ }^{2} \mathrm{~A}$ decline in sexual function among breast cancer patients has been found to be prevalent worldwide. ${ }^{3}$ Norley et al. found that $90 \%$ of Malaysian women with breast cancer experienced female sexual dysfunction (FSD). ${ }^{4}$ FSD refers to the disturbance in any phases of women's sexual response that leads to three disorders, namely female sexual arousal/interest disorder, female orgasmic disorder and genito-pelvic pain or penetration disorder. ${ }^{5}$ Boswell et al. contended that mastectomy, radiotherapy and chemotherapy were significant contributors to arousal, desire and orgasmic difficulties as well as lower body image scores, which in turn lead to sexual dysfunction. ${ }^{6}$ Unfortunately, only fewer than half of these women received treatment for FSD. ${ }^{6}$

Management of FSD is vital for women with breast cancer because of its devastating consequences, which include marital disharmony and a reduced quality of life. ${ }^{7}$ HCPs in breast cancer units should have basic knowledge about sexual health issues; they should be able to identify any changes in sexual function, discuss problems and address the need for sexual counseling or to provide referrals. $\underline{\underline{2}}$ Unfortunately, most of these issues are not recognized and are thus undertreated. $\underline{8} . \underline{\text { Canzona et al. }}$ 
conducted a qualitative study in 2018 among 36 oncology, gynecology, internal medicine and family medicine professionals. ${ }^{10}$ They found that HCPs' decisions to initiate sexual health discussions are often based on erroneous assumptions and practical constraints. ${ }^{10}$

In this paper, we explore HCPs' perceptions and experiences of sexuality and sexual dysfunction in managing women living with breast cancer using the Health Belief Model (HBM) and the phenomenological approach. The HBM model was adopted in order to understand the reasons for the HCPs' difficulties in this area. The model was theorized by social psychologists Rosenstock et al. in 1950 to assess individuals' beliefs about a disease and their choices regarding strategies to decrease its occurrence. 11 In this theoretical model, four perceptions play an important role: perceived seriousness, perceived susceptibility, perceived benefit and perceived behavior. Each of these perceptions, whether individually or in combination, can be used to explain the patients' and HCPs' health behaviors. $\underline{12}, \underline{13}$ Rosenstock et al. expanded the model by adding cues to action, motivating factors and self-efficacy. $\underline{\underline{14}}$ Culture, education level, past experiences and skills are also included. Self-efficacy refers to individuals' belief in their own ability to accomplish a task. $\frac{15}{}$ Self efficacy was added to the original four perceptions in the HBM (Figure 1). Individuals who believe that a new behavior is useful (perceived benefit) but do not think themselves capable of successfully adopting it (perceived barrier) are not likely to attempt it. ${ }^{16}$ The aim of this study to explore perceptions and experiences from HCPs in managing sexual dysfunction among breast cancer women in North East Malaysia.

\section{Method}

We adopted a qualitative design using the phenomenological approach for this study to understand the meanings and interpretations that Malay HCPs give to their behaviors within their cultural, social and religious context. ${ }^{17}$ This type of approach offers an opportunity to gain multifaceted, in-depth insight into participants' working experiences related to their way of discovering FSD and sexual health issues among women with breast cancer, how they converse with them and how they offer treatment. ${ }^{18}$ The dimensions of these experiences may also reflect beliefs, attitudes and behaviors that are difficult to measure in a quantitative way. ${ }^{19}$

Healthcare providers who work at two tertiary hospitals in North East Malaysia were purposively selected from hospital database lists. The participants had at least one year of experience in managing women with breast cancer. They were invited to participate via email. The number of participants in this study was determined by the saturation of data, whereby recruiting was stopped when little new data emerged. 20

In-depth interviews were conducted by the main author in Malay and were guided by a semi-structured questionnaire after obtaining the participants' consent to take part in the study. A pilot study was conducted on three HCPs with an experienced researcher. Before starting the interview session, a brief demographic form was provided for the interviewees to fill in. It included age, gender, duration of practice, 
area of practice, subspecialties and details of any training in women's sexual health, particularly FSD. The interview sessions were conducted mainly in a vacant room at the clinic and each interview lasted around one hour and was audiotaped.

The interviews were transcribed verbatim before being transferred into the NVivo (Qualitative Research Computer Analysis Package) software. The transcriptions were analyzed using thematic analysis. The data were familiarized through reading participants' transcriptions multiple times until meaning was derived from each paragraph. The initial codes were inserted, and axial codes were created. The magnitude, interaction of codes and conceptual thoughts that were relevant for the purpose of this study were highlighted. We revised interrelated and connected emergent sub-themes and grouped them under the main themes.

To certify rigors and trustworthiness, meticulous discussion was conducted to ensure the themes used fitted with existing codes. The peer review method was applied to ensure credibility of codes ad themes in this paper.

This study was conducted under the ethical approval of Universiti Sains Malaysia (USM/JEPeM/17090387) and Ministry of Health of Malaysia. (NMRR-18-373-39791(IIR)).

\section{Results}

Fifteen HCPs include six staff nurses, four medical officers, three specialists and two counsellors with the mean of 8.5 years experiences in managing breast cancer patients were involved in this study. The sociodemographic information of the participants interviewed is presented in Table 1. Three main issues were identified from HCPs regarding in handling sexual dysfunction: scarcity of knowledge, sociocultural influence and specialty-centric barriers (Table 2).

\section{SCARCITY OF KNOWLEDGE}

\section{Narrow meaning of sexuality}

Most of the HCPs described the meaning of sexuality as purely sexual intercourse. One viewed sexuality as body image or body ideals for both genders, male and female:

Sexuality... if we talk about sexuality, I imagine it as the general appearance of the individual according to their gender, men and women respectively. ( $\mathrm{Dr} \mathrm{H}$, oncologist, four years of experience)

$\operatorname{Dr} \mathrm{N}$, meanwhile, felt that sexuality should be broader in meaning, involving the emotions that couples experience together, their ability to perform gender roles as well as obligations in the relationship:

The meaning of sexuality is extensive, it is not focused on sexual intercourse per se, but more on the husband and wife relationship that results in marital happiness... also the image of the woman, whether 
she feels herself to be complete or incomplete. ( $\mathrm{Dr} \mathrm{N}$, surgeon, 11 years of experience)

\section{Unfamiliarity with FSD}

Almost all doctors remarked that their lack of exposure to FSD was due to unfamiliarity with the disorder and treatment because it was not covered in their training.

If they develop low self-esteem due to their body image, we can refer them to a plastic surgeon for reconstructive surgery or an implant... But I am not sure the treatments for FSD. (Mrs. K, oncology staff nurse, eight years of experience)

Dr S received complaints from a few patients regarding dryness but not sure how to treat it and refer them to the counsellor.

\section{Lack of training}

Lack of training in sexual health and FSD leads to a lower level of confidence in HCPs regarding discussing sexual problems with their patients. As stated earlier, most of them attributed not knowing about FSD to not having the proper training on this topic either during their undergraduate or postgraduate studies. In addition, several HCPs stated that they had never attended any related courses and FSD was never discussed in their continuous medical education program at the hospital level:

We do not have training about FSD and its management, just from our experiences from seeing the patient with sexual problems... I usually will spend time with them ...if needed, I refer them to relevant specialists. (Dr N, surgeon, 11 years of experience)

This is in contrast with junior HCPs, for whom sexual health and FSD were included in their undergraduate syllabus. However, knowledge without training caused them to have less experience in handling such patients:

We learn about FSD during medical school. But we rarely handle patients with sexual dysfunction. We saw erectile dysfunction cases; however, we did not know how to manage them. We did not have much exposure. (Dr I, oncologist, two years of experience)

\section{SOCIOCULTURAL INFLUENCES}

\section{Sex and privacy, reticence and embarrassment}

Many HCPs shared their difficulties in initiating conversations about sexual problems among women with breast cancer because culturally the topic is considered taboo.

Most patients refuse to talk about it... because sexual issues are too private for them. (Dr N, surgeon, 11 years of experience). 
This phenomenon has caused some HCPs to consider it to be offensive to talk about sex if their patients did not complain about it first. HCPs were also embarrassed to deal with sexual problems, as many confessed to being too shy to initiate and discuss FSD and sexual health. This made most HCPs keep their quiet and hope that patients would disclose their problem during the first encounter themselves. However, some realized that they are supposed to develop a rapport, take some time and make a move to start the conversation if they want these women to voluntarily to reveal their problems.

I am very shy. I force myself to ask...to provide treatment and save their marriage. I usually say sorry first before I ask about sexual issues because I want to make the conversation as neutral as I can and to develop trust in the patient. (MrsG oncology counselor, 13 years of experience)

The sensitivity of the issue in the community has caused a few HCPs to have trouble finding the appropriate words and ways to ask their patients about sexual problems in a manner that would ensure that patients would be less likely to feel upset.

\section{Sexual health is not the patient's priority}

In elaborating on the influence of culture on women with breast cancer, $\mathrm{Dr} \mathrm{H}$ realized that Malay culture is more entrenched in east coast states, particularly in suburban and rural areas:

When I was in Kota Kinabalu, most of my patients, including Malays, were concerned about body image and their sexual life... Maybe (they are from) the urban area. They were more open. In Kelantan, most Malays are wearing 'baju kurung', thus physical appearance is not important...maybe sex is not essential for them too. (Dr H, oncologist, four years of experience)

HCPs revealed that most patients under their care seemed more worried about their disease and the treatment's side effects.

They are more concerned about skin dryness and itchiness after radiotherapy. Even cervical cancer patients who receive radiotherapy for the pelvic area only complain about vaginal dryness rather than telling us about sexual dysfunction. (Dr I, oncology doctor, two years of experience)

HCPs also explained that their patients choose to turn to religion when they are sick because they believe illness to be a trial from God. Thus, it is thought to be a more appropriate time for patients to ask for healing of their illness as well as forgiveness:

They focus more on their family; the problem arises after they get cancer. And some patients said the important things for them now are to seek God's forgiveness and abide more frequently to religion. (Mrs A, surgery staff nurse, 11 years of experience)

These women choose to provide sexual satisfaction only for their spouse while they suffer from breast cancer and ignore their own satisfaction. 
When asked about sexual intercourse, patients said it is like before. When I asked about satisfaction, they kept quiet... They do it [sexual intercourse] just to give their spouse sexual satisfaction. For them, this is enough. (Dr E, psychiatrist, four years of experience).

They told us that their desire for sex was reduced and they were not involved in sexual intercourse as much compared with when they were younger (under 40 years). They said their husbands did not bother [them] much on that. (Dr L, oncologist, three years of experience)

\section{Sexual discussion and social status}

The appropriate social status is viewed as an important element in taking sexual history and having discussions on sexual health with women with breast cancer. Many HCPs realized that gender difference and being single prevented them from obtaining adequate sexual history from these patients since they felt less comfortable and embarrassed.

I have seen a conversation between a female patient and a male doctor. The doctor asked how her sexual relationship with her husband was. The patient started to cry and then was reluctant to talk further. The male doctor then offered her to see a female doctor. She agreed. (Mrs J, oncology staff nurse, four years of experience)

Age is another factor that is commonly considered as a cut-off point to discussing sexual health with patients. Many HCPs perceived sex as not important to elderly patients.

Sexuality for them... is more about spending their lives together, like touching and caressing each other, not sexual intercourse. (Mrs D, surgery staff nurse, five years of experience).

\section{SPECIALITY- CENTRIC}

\section{Low priority for sexual health}

Multidisciplinary approach is prudent for HCPs to manage their patients' problems in a holistic manner. They were more focused on treating the cancer itself and explaining the side effects of treatment.

In breast cancer, we only concentrate on her cancer. For sexual problems, we rarely ask because our main concern is to identify any complications from the chemodrugs. (Mrs C, oncology staff nurse, 19 years of experience).

I do not have much information on that because I rarely ask about sexual problems in my patients. I concentrate more on financial and emotional issues. (Mrs D, oncology counselor, 15 years of experience).

There were no referrals from other health disciplines to the psychiatrist for appropriate FSD treatment. I never get referral from other department for these problems. (Dr E, psychiatrist, four years of experience). 


\section{Time and privacy limitation.}

Limited resources and the overwhelming number of patients who visited oncology clinics lead to HCPs not having enough time and no privacy to screen for FSD:

We cover about 40 patients per day only in the morning session. We also get referrals from our ward if any problems arise with in-patients from other departments and from other hospitals. [...] More time is needed for complicated cases and we focus more on their disease rather than talking about sex. (Dr O, oncologist, two years of experience).

We shared two medical officers in one room, hence there is no privacy for the patient to discuss on sexual problems. \{Dr A, medical officer, two years of experiences).

Difficulty to spend a suitable time also acts as a barrier for HCPs to discuss sexual issues with their patients even though patients admit in the ward. The stage of the disease and the side-effects of medications also limit the conversations about sexual issues.

\section{Discussion}

The synthesis of the findings of this study is guided by the Health Belief Model, which theorizes the influence of modifying factors such as knowledge, social culture and working culture on HCPs' sexual health perceptions and management (Figure 2). The present study identified two types of HCPs: those with higher awareness of sexuality and sexual health (exposed HCPS) and those lacking such awareness (lack-of-exposure HCPs). The exposed HCPs had a better understanding of sexuality and sexual dysfunction. The lack-of-exposure HCPs, i.e. most of the HCPs, exhibited lower cues to action (Figure 3) because they were unsure about the definitions of sexuality and sexual dysfunction. Thus, a narrow definition of sexuality can influence HCPs' judgement about sexual health and FSD and their evaluation and management of their patients. This finding supports the findings of previous studies. $\underline{9}, \underline{10}, 21-23$

Training is another cue to action. The present study found the training and communication skills of HCPs regarding sexual health to be inadequate. Other studies have also found these phenomena to be the reasons for HCPs' lack of knowledge in addressing and treating their patients' sexual health concerns. $\underline{24-}$ $\underline{26}$ The lack of understanding of their role related to patients' sexual health also worsens their ability to address sexual problems. $\underline{27} \underline{\underline{28}}$ Furthermore, the lack of time and privacy in the clinical and ward settings creates a barrier to the appropriate management of sexual health concerns. The examination rooms are often shared and the wards tend to be shared open spaces as well. $\underline{29-31}$ Finally, the HCPs' ability to change personal attitudes about sexuality is also an important factor in their involvement in screening and managing sexual health. $\underline{32}$

The lack-of-exposure HCPs also held the opinion that their patients focused more on their disease than on their sexual health. Thus, the assumption was that Malay women were less likely to experience FSD. They believed that culture, religion and family influenced the patients' concerns about sexual health. In 
Malay culture, women often prioritize satisfying their families' and husbands' needs; thus, their sexual health is often neglected, and sexual issues are kept hidden. $\underline{33} \underline{34}$. This situation has led to HCPs' perception that communicating about sexual health and FSD is less beneficial and therefore not a priority in patient management. $\underline{21} \underline{23}, \underline{35}$

Embarrassment is another example of the perceived barriers for the lack-of-exposure HCPs, contributing to their and their patients' discomfort in discussing sexual issues. $.123, \underline{26}$ Embarrassment affects their ability to ask the appropriate questions and to think beyond the usual care in their efforts to improve the patients' quality of life. ${ }^{37}$ Instead, they tend to not consider the effects of the long treatment duration and ignore patients' sexual health until the onset of distress or depression. $\underline{21} \underline{\underline{38}}$

The differences between the doctors and the patients regarding age, marital status and gender

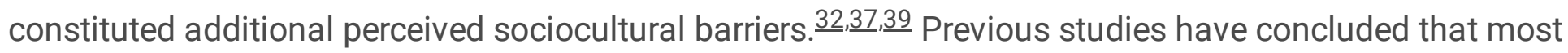
HCPs are uncomfortable about interviewing opposite-gender and elderly patients because of the patients' preferences for doctors of the same gender and a similar age. $\underline{29}, 39$ A survey in the United Kingdom found that public nurses preferred to have consultations about sexual issues with same-gender patients. ${ }^{40}$ Similar results were found for primary care specialists in Malaysia regarding questions about the patients' sexual issues. $\underline{21}$

Most of the lack-of-exposure HCPs, especially the medical officers and staff nurses, had limited time for managing their patients because they were more focused on completing the tasks related to the management of the cancer. Time was not a problem for the counselors and specialists; nevertheless, sexual issues were not likely to be discussed with the patients because of embarrassment and sociocultural factors. Only one exposed $H C P$ with a sexual training background had discussed sexual health with patients. Indeed, the HCPs with sufficient training, communication skills and knowledge about sexual issues did not consider time to be a significant barrier to communication about such issues. $\underline{22, \underline{36}}$

Thus, the lack-of-exposure HCPs perceived women with breast cancer as being unlikely to have sexual difficulties and FSD (lower perceived susceptibility). They also perceived these women to be less likely to have marital disharmony and psychosocial and health deterioration (lower perceived severity). This led them to believe that there was little benefit in evaluating the sexual health or FSD of their patients; consequently, fewer diagnoses and referrals were made in this regard. Finally, the HCPs' specialties and the time they required were also perceived as barriers to discussions about FSD. Ultimately, the findings

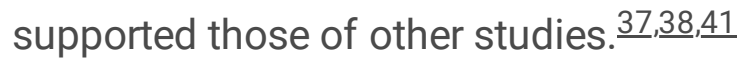

In contrast, the exposed HCPs perceived women with breast cancer as being at risk for FSD. Their colleagues' failure to make referrals was a perceived barrier and the reason for the low number of patients with FSD diagnoses. The lack of updated knowledge was another barrier. However, a few of the lack-of-exposure HCPs had greater insights, which improved their self-efficacy and allowed them to overcome erroneous understanding, attitudes and self-barriers. $\underline{42}$ Thus, the enhancement of HCPs' selfefficacy helps to reduce the perceived barriers. 
Self-efficacy is an important component for successful communication in order to reduce erroneous assumptions and negative influences among HCPs. It accounted for the individual differences in health behaviors. Self-efficacy is a powerful predictor of the intent to communicate about sexual health. $\underline{43}$ The findings indicate that individuals with self-efficacy are confident in their ability to communicate successfully about sexual issues.

The limitation of this study was the low participation rate, especially that of male HCPs. Most of the HCPs were female, married and Malay; thus, the views, experiences and perceived barriers of single male HCPs and other ethnic groups were not reflected. Further studies are needed to investigate the differences among HCPs in the Malaysian states regarding the management of FSD in women with breast cancer.

\section{Conclusion}

The HCPs need to acknowledge the prevalence of FSD and its impact on emotional and psychological care in women with breast cancer. Therefore, interventions to empower the knowledge, break the sociocultural barriers and improve the clinic settings are crucial for HCPs in managing FSD confidently.

\section{Declarations}

\section{Acknowledgement}

We would like to thank to all HCPs that participated in this study.

\section{Funding}

This study was funded by Universiti Sains Malaysia with Research University Grants (1001/PPSP/8012284) and Graduate Study Development Incentive Fund (TIPPS).

\section{Conflicts of interest}

The authors declare that they have no conflict of interest.

\section{Ethics approval:}

The study protocol and the questionnaires used in this study were reviewed and approved by the ethical approval of Universiti Sains Malaysia (USM/JEPeM/17090387) and Ministry of Health of Malaysia. (NMRR-18-373-39791(IIR)).

\section{Consent to participate:}

Patient information leaflets were distributed and written informed consent was obtained from the participants before they were recruited into the study. Participants were informed of any immediate results obtained from the study that might affect their care.

\section{Consent for publication:}


Patient information leaflets were distributed and written informed consent was obtained from the participants for publication.

\section{Availability of data and material:}

Data are kept at the Family Medicine Department, School of Medical Sciences, Universiti Sains Malaysia, 16150 Kubang Kerian, Kelantan, Malaysia. Data will be shared upon request and it is subjected to the data protection regulations.

Code availability: Not applicable.

\section{Authors' contributions:}

SBC, MMZ, and RDM conceptualized and designed the study. RDM and MMZ acquired the funding. RDM, LWY and PL supervised and coordinated the study. SBC, MMZ and RDM analysed and interpreted the data. SBC, MMZ and RDM drafted the manuscript. HMZ, LWY, and PL revised it critically for important intellectual content. All authors have read and given approval of the final manuscript. Each author has participated sufficiently in the work to take public responsibility for appropriate portions of the content as described above.

\section{References}

1. Abdullah NA, Wan Mahiyuddin WR, Muhammad NA, Ali ZM, Ibrahim L, Ibrahim Tamim NS, et al. Survival rate of breast cancer patients in Malaysia: a population-based study. Asian Pacific Journal of Cancer Prevention: APJCP [Internet]. 2013;14(8):4591-4. Available from: https://doi:10.7314/APJCP.2013.14.8.4591

2. Del Pup L, Villa P, Amar ID, Bottoni C, Scambia G. Approach to sexual dysfunction in women with cancer. International Journal of Gynecologic Cancer [Internet]. 2019:ijgc-2018-000096. Available from: https://doi: 10.1136/ijgc-2018-000096

3. Webber K, Mok K, Bennett B, Lloyd AR, Friedlander M, Juraskova I, et al. If I am in the mood, I enjoy it: an exploration of cancer-related fatigue and sexual functioning in women with breast cancer. The Oncologist [Internet]. 2011;16(9):1333-44. Available from: https://doi:10.1634/theoncologist.20110100

4. Norley S. Sexual dysfunction, body image distress and marital dissatisfaction in breast cancer patients of University Malaya Medical Centre (UMMC): University of Malaya; 2014.

5. IsHak WW, Tobia G. DSM-5 Changes in Diagnostic Criteria of Sexual Dysfunctions. Reprod Sys Sexual Disorders. 2013;2:122. doi:10.4172/2161-038X.1000122

6. Boswell EN, Dizon DS. Breast cancer and sexual function. Transl Androl Urol [Internet]. 2015;4(2):1608. Available from: https://doi:10.3978/j.issn.2223-4683.2014.12.04

7. Sbitti Y, Kadiri H, Essaidi I, Fadoukhair Z, Kharmoun S, Slimani K, et al. Breast cancer treatment and sexual dysfunction: Moroccan women's perception. BMC Women's Health [Internet]. 2011;11:29-. Available from: https://doi:10.1186/1472-6874-11-29. 
8. Park E, L. Norris R, Bober S. Sexual health communication during cancer care: barriers and recommendations. [Internet]. 2009;74-7. Available from:

https://doi:10.1097/PPO.0b013e31819587dc

9. Dyer K, das Nair R. Why don't healthcare professionals talk about sex? A systematic review of recent qualitative studies conducted in the United Kingdom. The Journal of Sexual Medicine [Internet]. 2013;10(11):2658-70. Available from: https://doi: 10.1111/j.1743-6109.2012.02856.x.

10. Canzona MR, Ledford CJW, Fisher CL, Garcia D, Raleigh M, Kalish VB. Clinician barriers to initiating sexual health conversations with breast cancer survivors: the influence of assumptions and situational constraints. Families, Systems, \& Health [Internet]. 2018;36(1):20-8. Available from: https://doi:10.1037/fsh0000307.

11. Rosenstock IM. Historical origins of the Health Belief Model. Health Education Monographs. 1974;2(4):328-35.

12. Shabibi P, Zavareh MSA, Sayehmiri K, Qorbani M, Safari O, Rastegarimehr B, et al. Effect of educational intervention based on the Health Belief Model on promoting self-care behaviors of type-2 diabetes patients. Electron Physician [Internet]. 2017;9(12):5960-8. Available from: https://doi:10.19082/5960

13. Brinsley KJ, Sinkowitz-Cochran RL, Cardo DM. Assessing motivation for physicians to prevent antimicrobial resistance in hospitalized children using the Health Belief Model as a framework. American Journal of Infection Control [Internet]. 2005;33(3):175-81. Available from: https://doi:10.1016/j.ajic.2004.12.004

14. Rosenstock IM, Strecher VJ, Becker MH. Social learning theory and the Health Belief Model. Health Education Quarterly. 1988;15(2):175-83.

15. Bandura A. Self-efficacy: the exercise of control. New York: Freeman; 1997.

16. Orji R, Vassileva J, Mandryk R. Towards an effective health interventions design: an extension of the health belief model. Online J Public Health Inform [Internet]. 2012;4(3):ojphi.v4i3.4321. Available from: https://doi:10.5210/ojphi.v4i3.4321.

17. Khan S. Qualitative research method - Phenomenology. 2014. 298-310 p.

18. Rasmussen DM, Hansen HP, Elverdam B. How cancer survivors experience their changed body encountering others. European Journal of Oncology Nursing [Internet]. 2010;14(2):154-9. Available from: https://doi:10.1016/j.ejon.2009.10.001

19. Hammarberg K, Kirkman M, de Lacey S. Qualitative research methods: when to use them and how to judge them. Human Reproduction [Internet]. 2016;31(3):498-501. Available from: https://doi:10.1093/humrep/dev334.

20. Saunders B, Sim J, Kingstone T, Baker S, Waterfield J, Bartlam B, et al. Saturation in qualitative research: exploring its conceptualization and operationalization. Qual Quant [Internet]. 2018;52(4):1893-907. Available from: https://doi:10.1007/s11135-017-0574-8

21. Muhamad R, Horey D, Liamputtong P, Low WY. Managing women with sexual dysfunction: difficulties experienced by Malaysian family physicians. Arch Sex Behav [Internet]. 2019;48(3):949-60. Available 
from: https://doi:10.1007/s10508-018-1236-1

22. Kingsberg SA, Schaffir J, Faught BM, Pinkerton JV, Parish SJ, Iglesia CB, et al. Female sexual health: barriers to optimal outcomes and a roadmap for improved patient-clinician communications. Journal of Women's Health [Internet]. 2019;28(4):432-43. Available from: https://doi:10.1089/jwh.2018.7352

23. Lai P, Yee Tan S, Liew S. Views and experiences of Malaysian family medicine trainees of female sexual dysfunction. [Internet]. 2016. Available from: https://doi:10.1007/s10508-016-0796-1

24. Ariffin F. Sexual history taking: assessing Malaysian medical student knowledge in sexual health. 2013.

25. Perz J, Ussher JM, Gilbert E. Constructions of sex and intimacy after cancer: a methodology study of people with cancer, their partners, and health professionals. BMC Cancer [Internet]. 2013;13(1):270. Available from: https://doi:10.1186/1471-2407-13-270

26. Hinchliff S, Gott M. Seeking medical help for sexual concerns in mid- and later life: a review of the literature. Journal of Sex Research [Internet]. 2011;48(2-3):106-17. Available from: https://doi:10.1080/00224499.2010.548610

27. Lieber E, Chin D, Li L, Rotheram-Borus MJ, Detels R, Wu Z, et al. Sociocultural contexts and communication about sex in China: informing HIV/STD prevention programs. AIDS Educ Prev [Internet]. 2009;21(5):415-29. Available from: https://doi: 10.1521/aeap.2009.21.5.415

28. Parish SJ, Hahn SR, Goldstein SW, Giraldi A, Kingsberg SA, Larkin L, et al. The international society for the study of women's sexual health process of care for the identification of sexual concerns and problems in women. Mayo Clinic Proceedings [Internet]. 2019;94(5):842-56. Available from: https://doi: 10.1016/j.mayocp.2017.11.002

29. Gott M, Hinchliff S. Barriers to seeking treatment for sexual problems in primary care: a qualitative study with older people. Fam Pract [Internet]. 2003;20(6):690-5. Available from: https://doi:10.1093/fampra/cmg612

30. Tudor KI, Eames S, Haslam C, Panicker J. Barriers in clinic to discussing sexual dysfunction due to multiple sclerosis. Journal of Neurology, Neurosurgery \& Psychiatry [Internet]. 2015;86(11):e4. Available from: https://doi.org/10.1136/jnnp-2015-312379.6

31. Bdair I, Constantino R. Barriers and promoting strategies to sexual health assessment for patients with coronary artery diseases in nursing practice: a literature review. [Internet]. 2017: 473-92. Available from: https://doi:10.4236/health.2017.93034

32. Wylie K. Assessment \& management of sexual problems in women. Journal of the Royal Society of Medicine [Internet]. 2007;100(12):547-50. Available from: https://doi:10.1258/jrsm.100.12.547

33. Sidi $\mathrm{H}$, Puteh SE, Abdullah N, Midin M. The prevalence of sexual dysfunction and potential risk factors that may impair sexual function in Malaysian women. The Journal of Sexual Medicine [Internet]. 2007;4(2):311-21. Available from: https://doi:10.1111/j.1743-6109.2006.00319.x

34. Mohdamin H. Budaya Melayu dan Ancaman. Majalah Dewan Budaya. 2013.

35. Mahmoud ZM, Fawaz MA. Nurse's perception of barriers toward discussing female sexual issues in nursing practice. Med J Cairo Univ. 2015;83(2):221-30. 
36. Abdulrasulnia M, Shewchuk RM, Roepke N, Granstaff US, Dean J, Foster JA, et al. Perceived barrier, practice patterns, and confidence among primary care physicians and gyanaecologists. Journal of Sexual Medicine [Internet]. 2010;7:2499-508. Available from: https://doi:10.1111/j.17436109.2010.01857.x

37. Gott M, Galena E, Hinchliff S, Elford H. "Opening a can of worms": GP and practice nurse barriers to talking about sexual health in primary care. Family Practice [Internet]. 2004;21:528-36. Available from: https://doi:10.1093/fampra/cmh509

38. Humphery S, Nazareth I. GPs view on their management of sexual dysfunction. Family Practice [Internet]. 2001;18(5):516-8. Available from: https://doi.org/10.1093/fampra/18.5.516

39. Burd ID, Nevadunsky N, Bachmann G. Original research-education: impact of physician gender on sexual history taking in a multispecialty practice. The Journal of Sexual Medicine [Internet]. 2006;3(2):194-200. Available from: https://doi:10.1111/j.1743-6109.2005.00168.x

40. Stokes T, Mears J. Sexual health and the practice nurse: a survey of reported practice and attitudes. The British Journal of Family Planning [Internet]. 2000;26(2):89-92. Available form: https://doi:10.1783/147118900101194328

41. Burd ID, Nevadunsky N, Bachmann G. Impact of physician gender on sexual history taking in a multispecialty practice. The Journal of Sexual Medicine [Internet]. 2006;3(2):194-200. Available from: https://doi:10.1111/j.1743-6109.2005.00168.x

42. Solursh DS, Ernst JL, Lewis RW, Prisant LM, Mills TM, Solursh LP, et al. The human sexuality education of physicians in North American medical schools. International Journal of Impotence Research [Internet]. 2003;15 Suppl 5:S41-5. Available from: https://doi:10.1038/sj.ijir.3901071

43. Hughes AK, Rostant OS, Curran PG. Improving sexual health communication between older women and their providers: how the Integrative Model of Behavioral Prediction can help. Research on Aging [Internet]. 2013;36(4):450-66. Available from: https://doi:10.1177/0164027513500055

\section{Tables}

Table 1: Profile of healthcare provider involved in study, $n=15$ 


\begin{tabular}{|lll|}
\hline Variables & Range Mean (SD) & $\mathrm{N}(\%)$ \\
\hline Age & $28-54$ & - \\
& $38.8(7.8)$ & \\
Male & & $-10)$ \\
Female & & $15(100)$ \\
\hline Year in practice & $2-19$ & - \\
& $8.5(5.2)$ & \\
\hline Job description & & $6(40 \%)$ \\
Staff Nurse & & $4(26.7 \%)$ \\
Medical Officer & & $3(20 \%)$ \\
Specialist & & $2(13.3 \%)$ \\
Counsellor & & $2(13.3 \%)$ \\
\hline Practice area & & $12(80 \%)$ \\
Surgery & & $1(6.6 \%)$ \\
Oncology & & \\
Psychiatry & & \\
\hline
\end{tabular}

Table 2 : Experiences and barriers in managing female sexual dysfunction among women with breast cancer 


\begin{tabular}{|c|c|c|}
\hline Themes & Subthemes & Axial Coding \\
\hline \multirow{8}{*}{$\begin{array}{l}\text { Scarcity of } \\
\text { knowledge }\end{array}$} & \multirow{2}{*}{$\begin{array}{l}\text { Narrowing meaning of } \\
\text { sexuality }\end{array}$} & Sexuality as sexual intercourse \\
\hline & & Sexuality as gender related body image \\
\hline & \multirow[t]{3}{*}{ Unfamiliarity with FSD } & . Inappropriate or incorrect definition \\
\hline & & - Unknowledgeable about screening method \\
\hline & & - Unsure about treatment \\
\hline & \multirow[t]{3}{*}{ No proper training } & No in-house training for FSD \\
\hline & & $\begin{array}{l}\text { Never attending outside course or updating } \\
\text { course on FSD / sexual health }\end{array}$ \\
\hline & & $\begin{array}{l}\text { FSD was not in their previous undergraduate or } \\
\text { postgraduate curriculum }\end{array}$ \\
\hline \multirow{10}{*}{$\begin{array}{l}\text { Sex and socio- } \\
\text { cultural } \\
\text { influences }\end{array}$} & \multirow[t]{4}{*}{$\begin{array}{l}\text { Sex is privacy, reticence } \\
\text { and embarrassment }\end{array}$} & $\begin{array}{l}\text { Talking about sex is culturally secret, only } \\
\text { between the couple }\end{array}$ \\
\hline & & Sex is a sensitive issue in community \\
\hline & & $\begin{array}{l}\text { Assume doctor-patient conversation about sexual } \\
\text { issues cause offence }\end{array}$ \\
\hline & & $\begin{array}{l}\text { Embarrass to initiate discussion of sexual health } \\
\text { unless volunteered by patients }\end{array}$ \\
\hline & \multirow[t]{4}{*}{$\begin{array}{l}\text { Sexual health is not } \\
\text { patients' priority }\end{array}$} & $\begin{array}{l}\text { Sex is not important in east coast and rural } \\
\text { population }\end{array}$ \\
\hline & & $\begin{array}{l}\text { Concern on the disease, treatment side effect, the } \\
\text { family matters and relationship with God }\end{array}$ \\
\hline & & Provide sexual satisfaction only for husband \\
\hline & & $\begin{array}{l}\text { Husband disregarded their sexual pleasure due to } \\
\text { empathy }\end{array}$ \\
\hline & \multirow[t]{2}{*}{$\begin{array}{l}\text { Sexual discussion and } \\
\text { social status }\end{array}$} & $\begin{array}{l}\text { Being single and gender difference hinder the } \\
\text { conversation }\end{array}$ \\
\hline & & Perceived sexuality in elderly is not important \\
\hline \multirow[t]{5}{*}{ Specialty-centric } & \multirow[t]{3}{*}{$\begin{array}{l}\text { Low priority for sexual } \\
\text { health }\end{array}$} & $\begin{array}{l}\text { Focused on breast cancer management, } \\
\text { misconception on multidisciplinary care }\end{array}$ \\
\hline & & - Felt sexual problem was not patients' big issues \\
\hline & & $\begin{array}{l}\text { Assumed patients had no risk to develop FSD as } \\
\text { it not involved genital area }\end{array}$ \\
\hline & \multirow[t]{2}{*}{ Limitation of time } & Limited resources with abundant of patients \\
\hline & & Difficulty to find suitable time \\
\hline
\end{tabular}


Figures

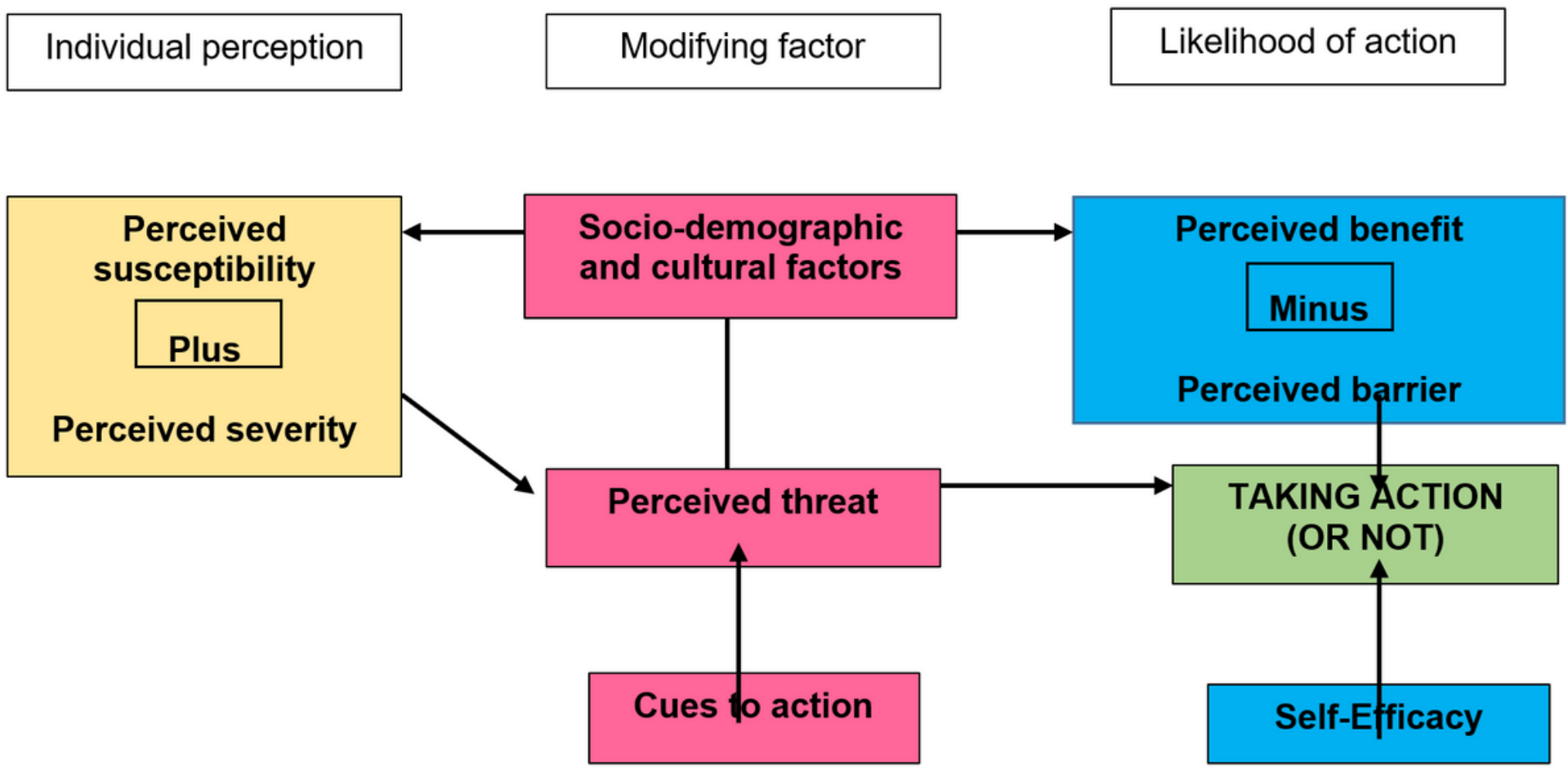

Figure 1

Health Belief Model (Adapted from Hayden, (2019)) 


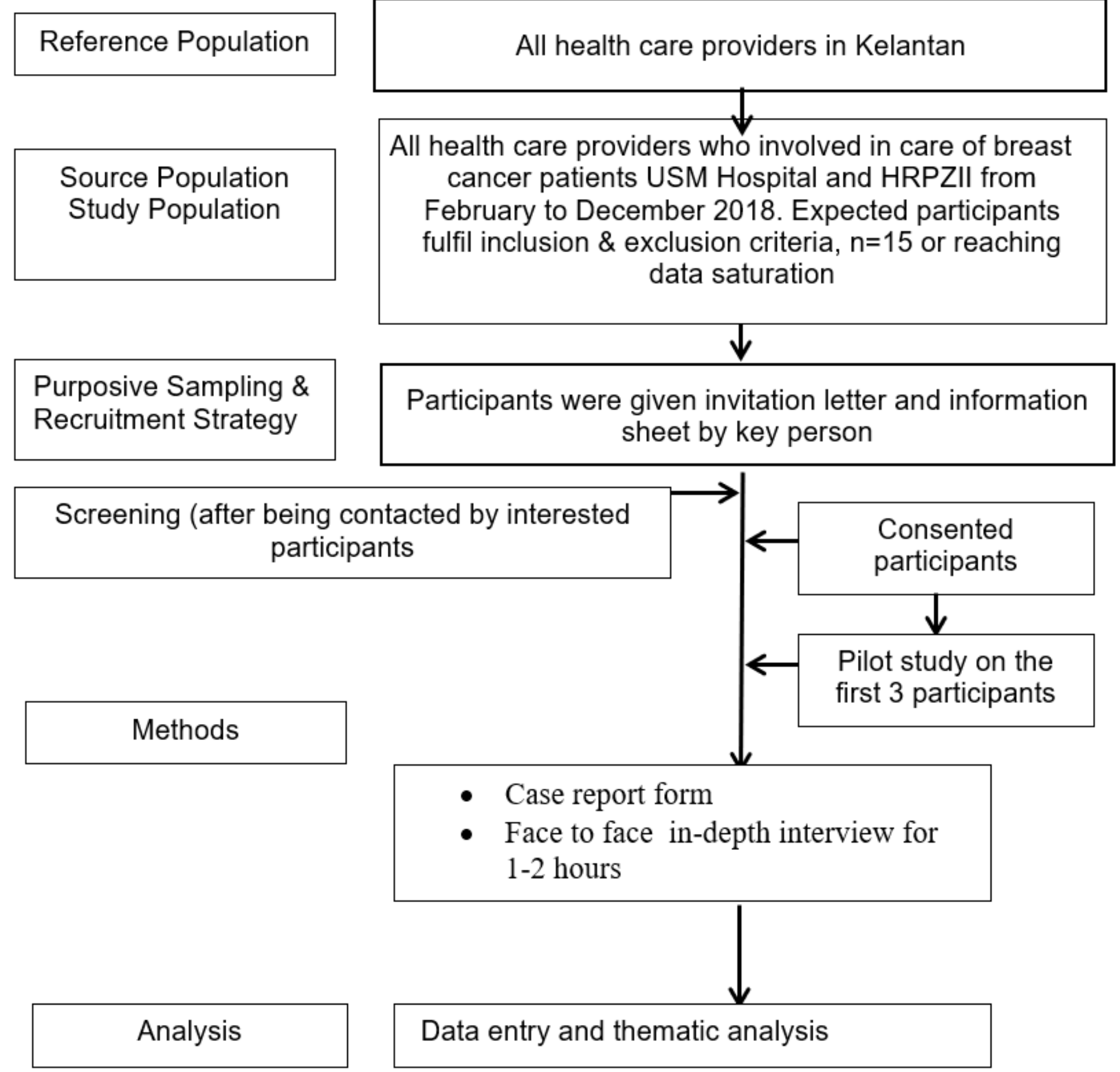

Figure 2

Flow chart for the study 


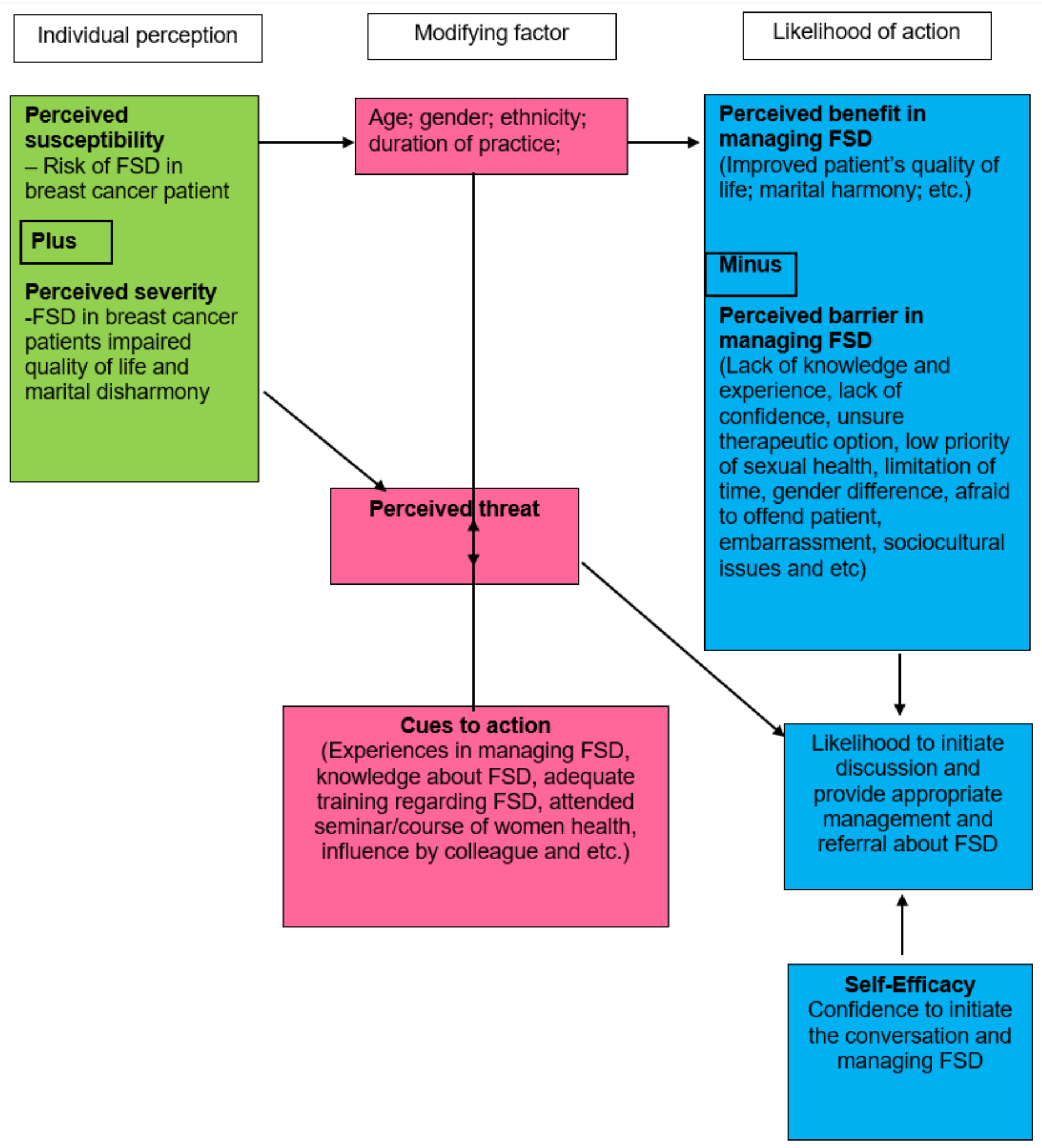

\section{Figure 3}

Barrier in Managing FSD according to HBM (Rosenstock et al., 1988) 